Agronomía Costarricense 43(2): 75-89. ISSN:0377-9424 / 2019

www.mag.go.cr/rev_agr/index.html www.cia.ucr.ac.cr

\title{
DETERMINACIÓN DE LA PRESENCIA DE CRISTALES DE OXALATO DE CALCIO ASOCIADOS A LA ENFERMEDAD ABIÓTICA CONOCIDA COMO "MANCHA BLANCA" EN Hylocereus costaricensis
}

\author{
Kenneth Retana-Sánchez ${ }^{1 / *}$, Oscar Castro-Zúñiga ${ }^{* *}$, Mónica Blanco-Meneses ${ }^{* * *}$ \\ Palabras clave: Hylocereus costaricensis; mancha blanca; acumulación; recubrimiento; oxalatos de calcio. \\ Keywords: Hylocereus costaricensis; white spot; accumulation; coating; calcium oxalates. \\ Recibido: 04/05/18 \\ Aceptado: 07/09/18
}

\begin{abstract}
RESUMEN
En un estudio del diagnóstico de las enfermedades presentes en el cultivo de la pitahaya (Hylocereus costaricensis), realizado en el periodo 2011-2013, se observó un recubrimiento amarillento endurecido sobre la superficie de tejido joven, el cual avanzó de los bordes hacia el centro del tallo; posteriormente mostró una coloración blanquecina-grisácea, principalmente en cladodios maduros. Se determinó la presencia de cristales de oxalato de calcio asociados a estos síntomas mediante el análisis de tejido afectado por microscopía electrónica, espectrometría de rayos $\mathrm{X}$ y difracción de rayos $\mathrm{X}$. Según el análisis foliar, se obtuvo $147 \%$ más calcio en cladodios sintomáticos en comparación con tallos sanos. A través del microscopio electrónico se observaron cristales con forma de rafidio y cubos en material afectado, compuestos de carbono, oxígeno y calcio, principalmente. Mediante la difracción de rayos $\mathrm{X}$ se confirmó la presencia de oxalatos en

1 Autor para correspondencia. Correo electrónico: kretana@uned.ac.cr

* Universidad Estatal a Distancia, Cátedra de Ciencias Agropecuarias, Costa Rica.

(iD) 0000-0003-1937-7249.

** Universidad de Costa Rica, Facultad de Ciencias Agroalimentarias, Centro de Investigaciones en Protección de Cultivos, Costa Rica.

(iD) 0000-0002-5999-5749.
\end{abstract}

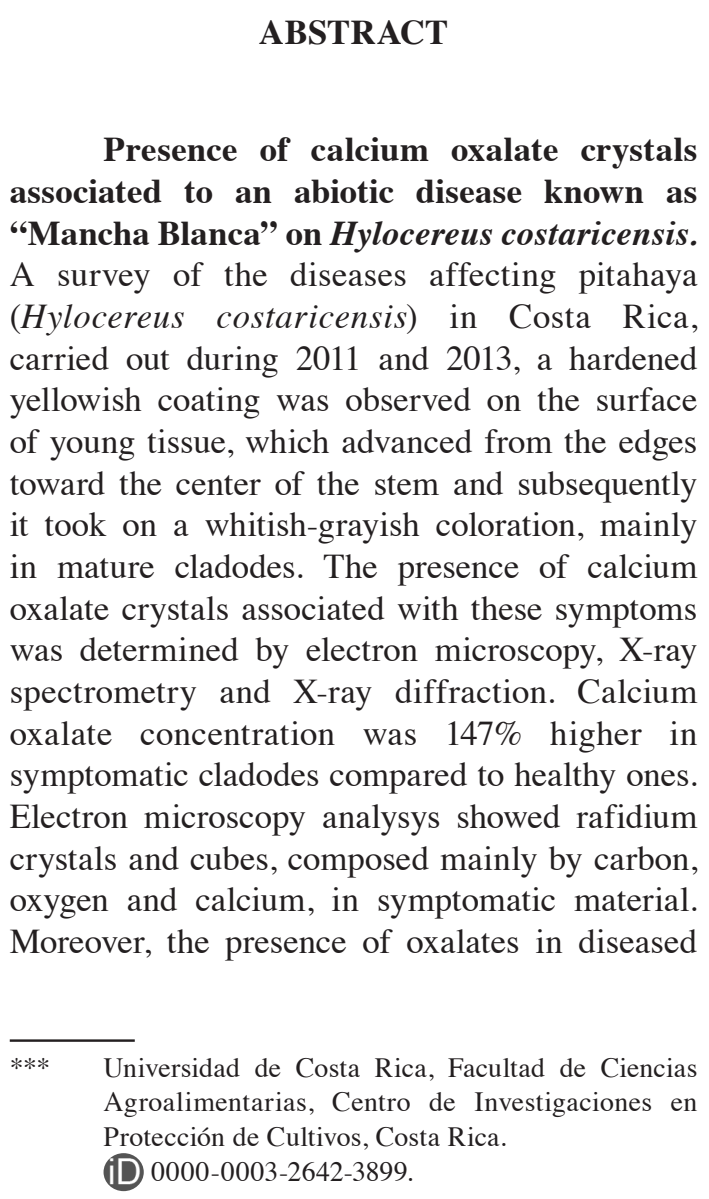

ABSTRACT 
tejido enfermo. En esta investigación se determinó la presencia de cristales de oxalatos de calcio asociados a la enfermedad abiótica "mancha blanca" que afecta la capacidad fotosintética de las plantas de Hylocereus costaricensis en Costa Rica.

\section{INTRODUCCIÓN}

El fruto de la pitahaya (Hylocereus spp.) es un recurso que se cultiva en zonas tropicales y subtropicales relativamente intensiva. La producción se comercializa sobre todo en los mercados regionales de los países productores, pero también se coloca con éxito en el mercado internacional de frutos exóticos, como fruto fresco, aunque también en forma de pulpa congelada o productos procesados (Vargas et al. 2005).

En Europa y Estados Unidos se ha incrementado el interés en este fruto por su apariencia exótica y porque es rico en nutrientes, además, presenta beneficios para la salud debido a sus propiedades nutracéuticas. Esto ha fomentado que en Costa Rica se desarrollen diferentes líneas de investigación con el objetivo de conocer los aspectos agronómicos y productivos alrededor de este cultivo (Dembitsky et al. 2011, Tenore et al. 2012).

Las enfermedades que afectan a esta planta, han sido estudiadas desde hace seis años para determinar los agentes causales asociados. Una de las patologías de carácter abiótico que se presentan en este cultivo y de la cual se tiene poco conocimiento es la mancha blanca que recubre la epidermis de los tallos. Es posible que asociada a esta, se encuentran cristales de oxalatos de calcio $\left(\mathrm{Ca}_{2} \mathrm{C}_{2} \mathrm{O}_{4}\right.$ o $\left.\mathrm{CaOx}\right)$ (Retana 2015), que es común en las cactáceas.

Se han encontrado estos cristales en células de la epidermis de 245 especies de cactus (Hartl et al. 2007). Asimismo, Viñas (2011) menciona la presencia de $\mathrm{Ca}_{2} \mathrm{C}_{2} \mathrm{O}_{4}$ dentro de células de los tallos de 2 genotipos de pitahaya (Orejona y San Ignacio), que se cultivan en tissue was confirmed by X-ray diffraction. In this research was determined the presence of calcium oxalate crystals associated to the "white spot" abiotic disease that affects the photosynthetic capacity of Hylocereus costaricensis plants in Costa Rica.

Costa Rica. También se ha encontrado sílice cristalino $\left(\mathrm{SiO}_{2}\right)$ en algunas especies de cactus (Monje y Baran 2000).

Generalmente dichos cristales se forman dentro de la membrana intravacuolar o cámara cristal de células especializadas llamadas idioblastos. En algunos casos se forman fuera, pero muy cerca de esta organela. Existen proteínas, como la calsequestrina, que regulan la actividad del calcio citosólico antes de ser almacenado en las vacuolas (Franceschi y Horner 1980, Webb 1999, Franceschi et al. 1993, Faheed et al. 2012).

El precursor primario para la formación de los oxalatos es el ácido ascórbico, a partir del cual se forma ácido oxálico. Cuando las concentraciones de esta sustancia son altas, se activa la enzima oxalato oxidasa, proteína que actúa como regulador de ácido oxálico y participa en la formación de los cristales de oxalato (Keates et al. 2000, Kostman et al. 2001, Volk et al. 2002).

Se especula que en cactus "primitivos" los oxalatos de calcio se forman a partir del metabolismo CAM y se depositan como cristales monohidratados (whewellita), que es la forma más estable, con el objetivo de remover los excesos de iones $\mathrm{Ca}^{+2}$ de tejidos susceptibles. Algunos cactus que se han especializado conservan los oxalatos de calcio en el estado menos estable (dihidratados, wedellita) para extraer $\mathrm{Ca}^{+2}$, en caso de ser necesario (Volk et al. 2002, Monje y Baran 2002, Hartl et al. 2007, Monje y Baran 2010).

A pesar de que el calcio es necesario para la regulación de procesos dentro de la célula y es un componente esencial de las paredes de esta, no se han dilucidado por completo los propósitos de almacenar calcio en forma de oxalatos y la función que cumplen estos (Hartl et al. 2007). A la 
fecha no se registran reportes de deposiciones de calcio externas sobre la epidermis de los cactus, por esta razón, el objetivo del presente trabajo fue verificar la presencia de oxalatos de calcio en el recubrimiento que afecta los cladodios de Hylocereus costaricensis.

\section{MATERIALES Y MÉTODOS}

\section{Recolección de muestras}

La investigación se realizó en la finca "El Brillante", la cual se dedica a la producción de pitahaya orgánica y se ubica en Miramar de Puntarenas $\left(10^{\circ} 6\right.$ ' $14^{\prime \prime}$ latitud norte y $84^{\circ} 48^{\prime} 49^{\prime \prime}$ 'longitud oeste), Costa Rica. La población en el área de estudio fue de 2666 plantas.ha $^{-1}$, de las cuales se seleccionó 992 plantas distribuidas en 38 hileras para realizar este trabajo.

En cada una de las hileras, se observaron los diferentes síntomas presentes en los cladodios y se tomaron 50 muestras con tallos completos; cada una se envolvió en papel periódico humedecido y luego fueron colocadas en una bolsa plástica junto con la identificación correspondiente. El material que se recolectó se llevó al Laboratorio de Fitopatología del Centro de Investigación en Protección de Cultivos (CIPROC) de la Universidad de Costa Rica (UCR), para el respectivo análisis.

\section{Seguimiento de síntomas}

Debido a la diversidad de síntomas observados en la plantación se procedió a realizar un control de su evolución en el campo, a través del tiempo. Con ese propósito se marcaron 50 plantas con diferentes síntomas en la plantación y se procedió al control visual mediante fotografías generadas cada 15 días durante un periodo de un año con el objetivo de observar los cambios morfológicos, de tamaño y de coloración en el material sujeto a estudio.

\section{Caracterización de síntomas}

Se realizó la descripción de los síntomas en las 50 muestras recolectadas, con base en las características observadas en cada una de las partes valoradas que fueron textura, color, ubicación del síntoma en el tallo y facilidad de desprendimiento por raspado. Esto se hizo con ayuda de un estereoscopio.

\section{Aislamientos y cámara húmeda}

Se realizó el aislamiento de los síntomas presentes en 20 muestras mediante la metodología descrita por French y Hebert (1980) para hongos y bacterias. La desinfección del material se llevó a cabo con etanol al $70 \%$ e hipoclorito de sodio al $1 \%$ durante 50 y 20 segundos, respectivamente.

Los medios de cultivo utilizados fueron agar-papa-dextrosa al 1,5\% (PDA) y V8 (Atlas 2010). Se rasgó el tejido de manera longitudinal y se extrajeron segmentos de la zona de avance en la parte interna del mismo tanto en la parte afectada como en la parte sana donde se presentaron síntomas. Se realizaron 2 repeticiones por cada medio utilizado para un total de 80 aislamientos que fueron incubados en una cámara a temperatura de $25-30^{\circ} \mathrm{C}$ y en oscuridad por un periodo de 7 días.

Paralelamente, se colocaron 5 segmentos de tejido sintomático en una cámara húmeda y posteriormente se realizaron evaluaciones de los cambios registrados en el material, durante 2 semanas.

\section{Análisis foliar}

Se hizo un análisis foliar, químico completo de una muestra compuesta de tejido sintomático, obtenido de 4 cladodios y otra de tallos sanos que se llevó a cabo mediante la digestión húmeda con $\mathrm{HNO}_{3}$ y determinación por Espectrometría de Emisión Atómica con Plasma (ICP), bajo la certificación CIA-SC09-01-01-P10. El análisis se llevó a cabo en el Laboratorio de Suelos y Foliares de la UCR.

\section{Análisis mediante microscopía electrónica}

El análisis de tejido sintomático se efectuó con microscopía electrónica de barrido en el 
Centro de Investigación en Estructuras Microscópicas (CIEMIC) de la UCR; se recolectó 1 $\mathrm{cm}^{2}$ de 2 muestras de tejido sintomático, una con síntomas iniciales y otra con síntomas avanzados de la enfermedad y se fijaron en reactivo de Karnovsky al 2,5\% por 24 horas. Posteriormente, se lavaron 3 veces por 10 min con buffer de fosfatos de sodio $(0,1 \mathrm{M}, \mathrm{pH} 7,4)$ y luego se posfijaron en tetraóxido de osmio $\left(\mathrm{OsO}_{4}\right)$ al $1 \%$ en un buffer de fosfatos de sodio, aproximadamente por 1 hora, y se aplicaron 3 lavados de $15 \mathrm{~min}$ con agua destilada.

Posteriormente, se llevó acabo la deshidratación de las muestras mediante un lavado con etanol en concentraciones ascendentes: $30 \%$, $50 \%, 70 \%, 80 \%, 90 \%, 95 \%$ y 2 lavados al $100 \%$ durante 10 min cada uno. Luego, se sometieron a 4 inmersiones de 15 min en alcohol terbutílico (Mallinckrodt) y después se secaron por medio de un sublimador.

Las muestras se colocaron en bases de aluminio de $15 \mathrm{~mm}$, por medio de cinta de carbono de doble cara adhesiva, con puntos de contacto por medio de pintura de plata. Las muestras se recubrieron con aproximadamente $40 \mathrm{~nm}$ de metal $\mathrm{Au} / \mathrm{Pd}$ con un cobertor iónico; las bases de aluminio con las muestras se observaron en el microscopio electrónico de barrido, el cual opera a $15 \mathrm{KV}$.

\section{Determinación de la morfología de los cristales}

Se determinó la morfología de los cristales presentes en cada una de las muestras mediante la observación de estos en el microscopio electrónico de barrido. Estas se compararon con las morfologías encontradas por Hartl et al. (2007) en diferentes géneros de cactáceas, entre ellas Hylocereus, las cuales son: en el caso del estado monohidratado: romboidal, prismática, de barra (estiloide y rafidio), pinacoidal, agregado esférico (drusas) y transiciones entre estos; y en estado dihidratado: prisma tetragonal, piramidal tetragonal, bipiramidal tetragonal y pinacoidal basal.

\section{Análisis mediante espectrometría de rayos $\mathrm{X}$}

Se realizó el análisis de tejido sintomático con espectrometría de rayos $\mathrm{X}$ en el Centro de Investigación en Estructuras Microscópicas, UCR. Para esto se tomó $1 \mathrm{~cm}^{2}$ de 2 muestras de tejido sintomático, una con síntomas iniciales y otra con síntomas avanzados de la enfermedad. Las muestras se dejaron secar y luego se colocaron en bases de aluminio de $15 \mathrm{~mm}$ por medio de cinta de carbono de doble cara adhesiva. Las bases de aluminio con cada muestra se observaron en el microscopio electrónico de barrido, con sonda de rayos $\mathrm{X}$ incorporado, el cual opera a $15 \mathrm{KV}$.

\section{Análisis mediante difracción de rayos $\mathbf{X}$}

Se realizó el análisis de tejido sintomático con difracción de rayos X en la Unidad de Difracción de Rayos X de la Escuela de Química, UCR. Esto se realizó con material obtenido del raspado del recubrimiento presente en 6 cladodios con síntomas intermedios y 6 cladodios con síntomas avanzados de la enfermedad.

Se eliminó la materia orgánica presente en las muestras en hipoclorito de sodio al $6 \%$ por 24 horas. Posteriormente, se les realizó varios lavados con etanol al $70 \%$ y se filtró el producto final.

El material seco se analizó con difracción de rayos X. Esto se llevó a cabo con un equipo de difracción de polvos D8 Advance. Se utilizó una fuente de Cuk $\alpha 1-\mathrm{k} \alpha 2$, con configuración Bragg Bentano y detector lineal Lynx-eye. Se realizaron barridos en 20 de $5^{\circ}$ a $60^{\circ}$ con un incremento de $0,019^{\circ}$ por paso y una velocidad por paso equivalente de $334 \mathrm{~s}$.

Se hizo uso de la base de datos de difracción de rayos-x en polvos International Centre for Diffraction Data (Kabekkodu 2007) para la comparación e identificación de los minerales presentes en las muestras.

\section{RESULTADOS Y DISCUSIÓN}

Los resultados obtenidos de las pruebas anteriores, se relacionaron con la fisiología de la 
planta y con aspectos de carácter patológico asociados a la misma. Además, se analizó la interacción de estos resultados y los patógenos presentes en el sitio de estudio.

En evaluaciones preliminares de las enfermedades asociadas al cultivo de la pitahaya se observó sobre la superficie de tallos un recubrimiento de color blanco-grisáceo que avanzó hasta cubrir por completo los cladodios. Durante el desarrollo del estudio se determinó que estados avanzados del síntoma se presentan únicamente en tejido maduro.
Con el seguimiento de los síntomas se logró identificar que la enfermedad inicia como una sustancia amarillenta endurecida que brota de las areolas, que luego se extiende de los bordes de los tallos y progresa hacia el centro de los cladodios (Figura 1A). Con el paso del tiempo, el recubrimiento adquiere una tonalidad blanquecina (Figura 1B) hasta que en estados avanzados se torna completamente blanco-grisáceo (Figura 1C y 1D) y cubre completamente el tallo (Figura $1 \mathrm{E}$ y $1 \mathrm{~F})$. Dicho revestimiento, tanto en etapas iniciales como finales, se puede desprender si se le realiza un raspado. 

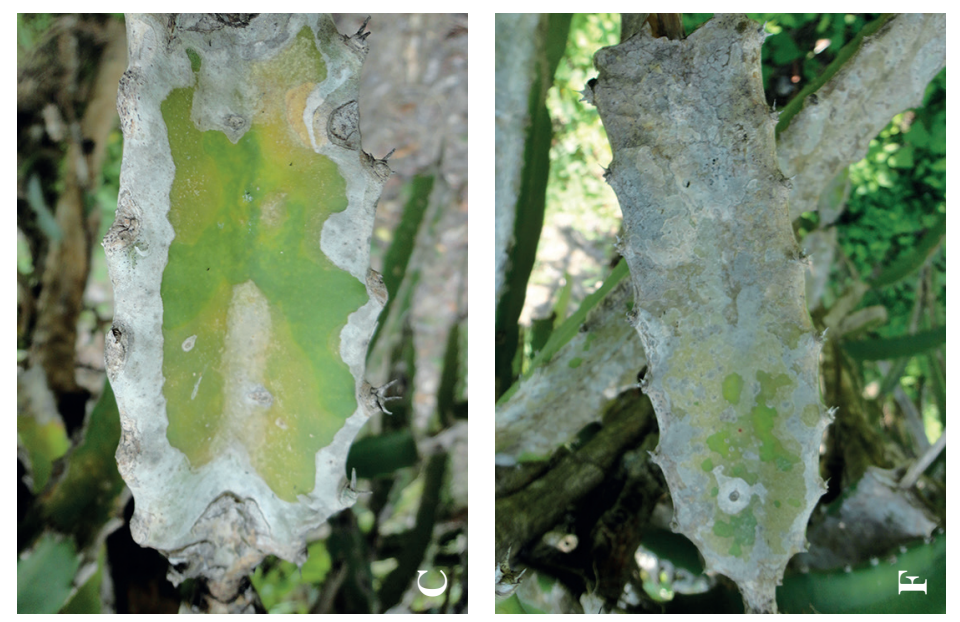

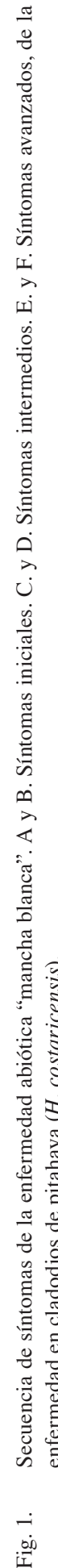
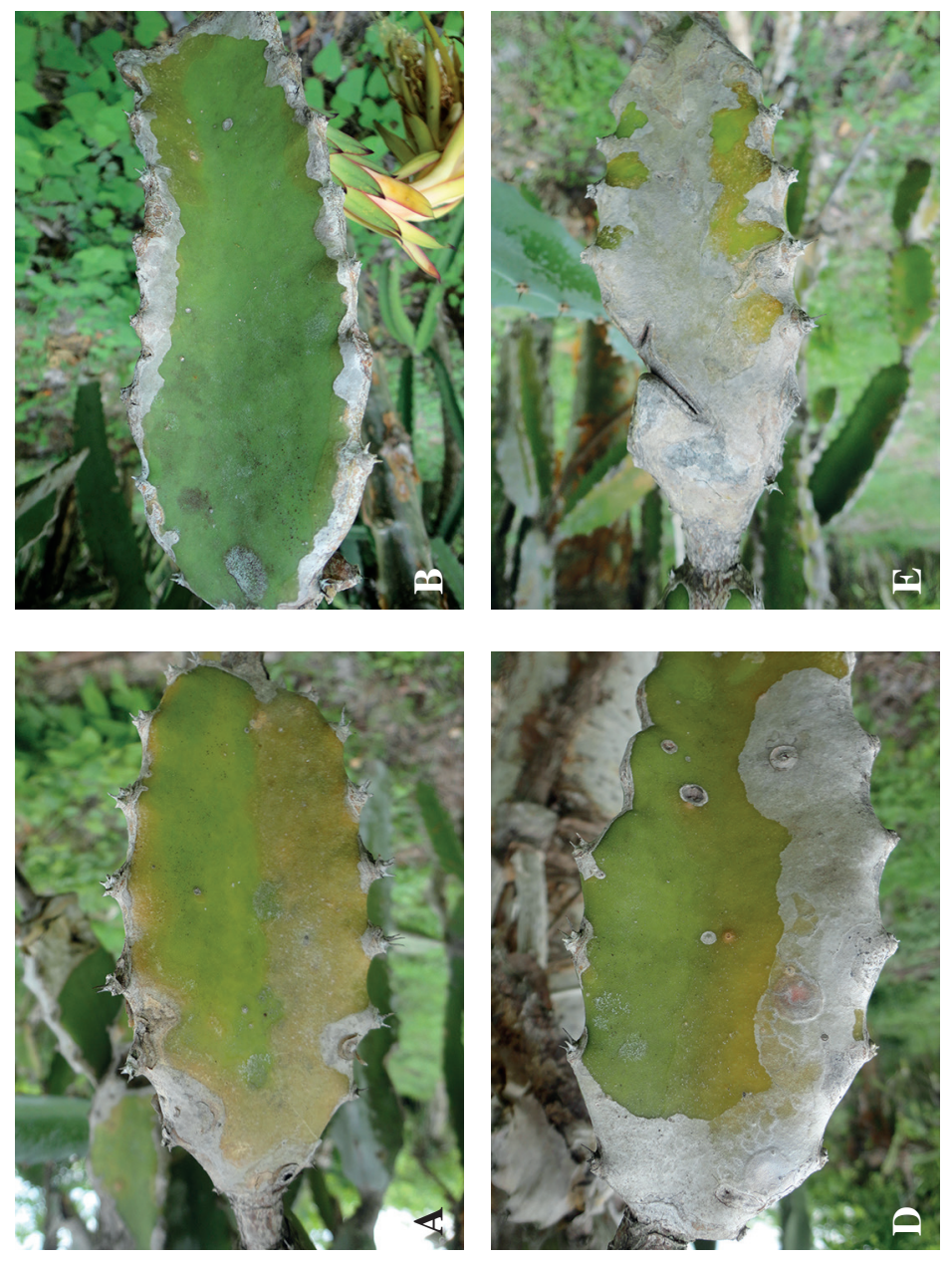

Agronomía Costarricense 43(2): 75-89. ISSN:0377-9424 / 2019 
En los aislamientos realizados no se presentó el crecimiento de microorganismos. Los resultados de la cámara húmeda fueron similares, debido a que no se obtuvieron signos de algún organismo presente en los tejidos analizados. Lo anterior, permitió asociar los síntomas con factores de carácter abiótico.

De acuerdo con el análisis foliar, el tejido afectado presentó $4,39 \%$ y el sano $1,78 \%$ de calcio, es decir, se obtuvo $147 \%$ más de este elemento en material sintomático en comparación con el sano (Cuadro 1). Juárez et al. (2012) reportan que los porcentajes de calcio presentes en el interior de los tallos de pitahaya se encuentran en el rango de 0,43 - 0,48\% mientras que en esta investigación se evidenció que las plantas en estudio absorben más calcio, esto es $1,3 \%$, posiblemente porque en el suelo hay un alto contenido de ese mineral (Cuadro 2).

Cuadro 1. Análisis químico foliar del tejido sano y enfermo de pitahaya (H. costaricensis).

\begin{tabular}{lcccccc|ccccc}
\hline & \multicolumn{9}{c}{} & \multicolumn{3}{c}{$\%$} & \multicolumn{4}{c}{$\mathrm{mg}^{-k g^{-1}}$} \\
Muestra & $\mathrm{N}$ & $\mathrm{P}$ & $\mathrm{Ca}$ & $\mathrm{Mg}$ & $\mathrm{K}$ & $\mathrm{S}$ & $\mathrm{Fe}$ & $\mathrm{Cu}$ & $\mathrm{Zn}$ & $\mathrm{Mn}$ & $\mathrm{B}$ \\
\hline Sin síntomas & 1,36 & 0,10 & 1,78 & 0,73 & 2,59 & 0,10 & 28 & 4 & 24 & 98 & 15 \\
Con síntomas & 0,54 & 0,03 & 4,39 & 1,23 & 1,83 & 0,12 & 49 & 4 & 27 & 107 & 18 \\
Diferencia (\%) & -60 & -70 & 1,47 & 68 & -29 & 20 & 75 & - & 13 & 9 & 20 \\
\hline
\end{tabular}

Fuente: Laboratorio de Suelos y Foliares de la Universidad de Costa Rica, 2014.

Cuadro 2. Contenido de calcio en el suelo proveniente de la plantación de pitahaya (H. costaricensis), Miramar, Puntarenas.

\begin{tabular}{lcccc}
\hline & Análisis químico de suelos & & \\
\hline Solución Extractora: & $\mathrm{pH}$ & $\mathrm{cmol}_{(+) .1^{-1}}$ & $\mathrm{Ca}$ \\
KCl-Olsen Modificado & $\mathrm{H}_{2} \mathrm{O}$ & ACIDEZ & 4 \\
Nivel crítico & 5,5 & 0,5 & 9,05 \\
Suelo analizado & 5,5 & 0,41 & \\
\hline
\end{tabular}

Fuente: Laboratorio de Suelos y Foliares de la Universidad de Costa Rica. 2014.

La diferencia en contenido de calcio entre el tejido sintomático y el tejido sano $(2,6 \%)$ indicó que este elemento es un componente abundante en el recubrimiento sobre la epidermis de este cactus. Otro elemento presente en dicho revestimiento, fue el silicio, en análisis foliares previos se obtuvo 1790 ppm de este elemento en cladodios sintomáticos y 1045 ppm en tallos sanos.

Se encontraron diferencias en los porcentajes de otros elementos analizados en tejido sano y enfermo. En este último se obtuvo un $60 \%$ y $39 \%$ menos de nitrógeno y potasio en comparación con los tallos sanos analizados (Cuadro 1). Probablemente, esto se debió a que, durante el periodo en que se realizó el estudio 2011-2013, se llevaron a cabo en la plantación algunas aplicaciones foliares de fertilizante con alta concentración de elementos que posiblemente recubrieron los cladodios enfermos y no permitió la absorción foliar; además vale señalar que los elementos mencionados son móviles dentro de la planta, por lo que se encuentran en mayor concentración en tallos jóvenes. Las muestras para el análisis de material sintomático se tomaron de cladodios 
maduros, debido a que la mancha blanca, como se mencionó, se presenta principalmente en tejido viejo. Lo anterior, sugiere una explicación de las diferencias descritas.

Según lo observado en las Figuras 2 y 3 se expone la presencia de cristales en tejido enfermo, tanto con síntomas iniciales como en estados avanzados. De acuerdo con su forma y al espectro obtenido por espectrometría de rayos $\mathrm{X}$, se determinó que se componen de oxalato de calcio, que es un compuesto frecuente en cactáceas, ya que se ha encontrado en 8 especies pertenecientes al género Hylocereus (Monje y Baran 2002, Hartl et al. 2007).
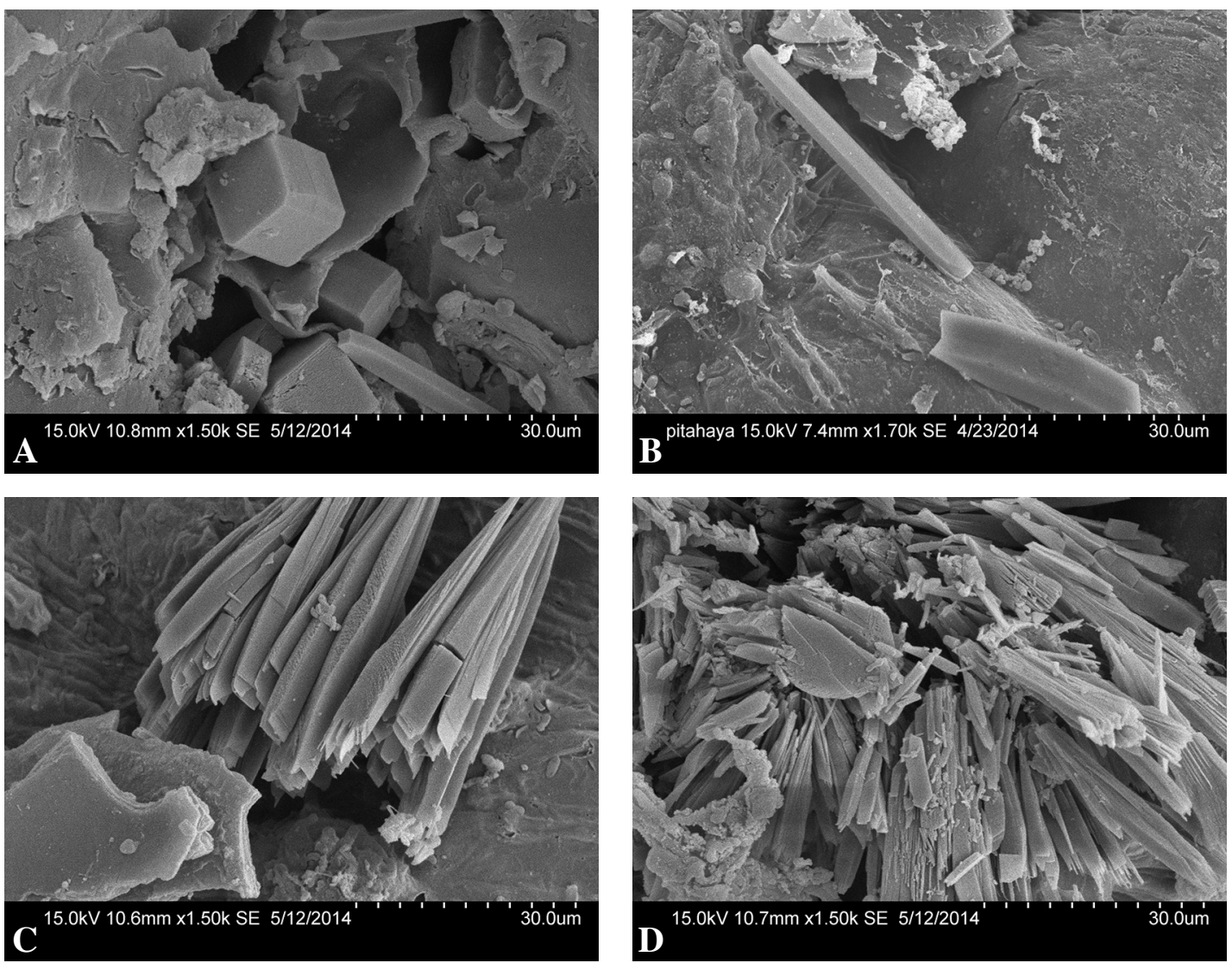

Fig. 2. Ultraestructura de los cristales de oxalato de calcio presentes en los tallos de pitahaya (H.costaricensis) con síntomas iniciales de la enfermedad. A. Morfología romboidal. B. y C. Morfología estiloide. D. Morfología de rafidio. 

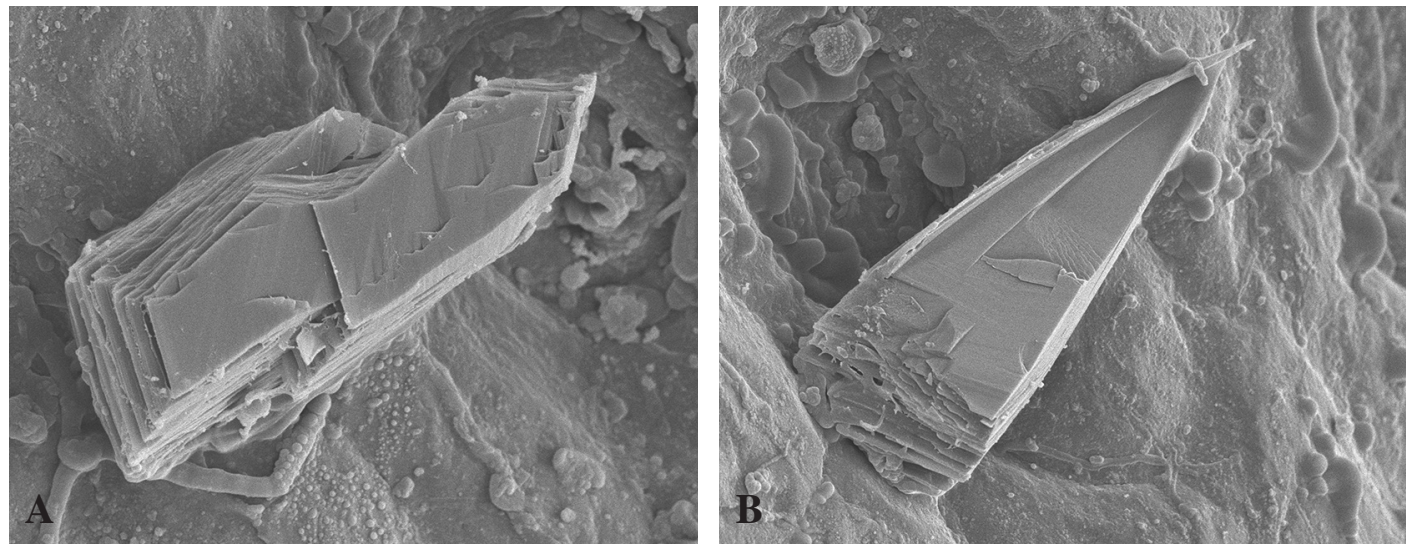

Fig. 3. Ultraestructura de los cristales de oxalato de calcio presentes en tallos de pitahaya (H. costaricensis) con síntomas avanzados de la enfermedad. A y B. Morfología pinacoidal.

Viñas (2011) observó cristales de este compuesto en tejido interno de 3 genotipos de H. costaricensis (Orejona, Cebra y San Ignacio) cultivados en la misma finca donde se realizó la presente investigación.

A partir de los análisis por espectrometría de rayos $\mathrm{X}$ realizados en diferentes cristales, se logró identificar un ámbito de concentración de $16 \%$ a $72 \%$ de $\mathrm{Ca}$ (Cuadro 3). Estas diferencias en el contenido del elemento, se pueden deber a la cantidad de iones disponibles, a procesos que ocurren a lo interno de la planta y a factores ambientales involucrados en la formación de los cristales (Franceschi y Horner 1980, Monje y Baran 2002). Otro elemento detectado en los cristales fue el silicio en concentraciones menores a un $4 \%$, por lo que no se consideró como un factor causal primario asociado a la enfermedad (Cuadro 3).

Cuadro 3. Análisis químico por espectrometría de rayos X de los cristales presentes en los tallos de pitahaya (H.costaricensis), con estados iniciales y avanzados del recubrimiento.

\begin{tabular}{ccc}
\hline Elemento & Intensidad (c/s) & Concentración (\%) \\
\hline C & $0,04-0,14$ & $1,3-35,8$ \\
O & $0,09-0,45$ & $26,2-66,9$ \\
$\mathrm{Ca}$ & $0,11-1,8$ & $16,1-71,7$ \\
$\mathrm{Si}$ & $0-0,22$ & $1,0-3,5$ \\
\hline
\end{tabular}

Fuente: CIEMIC, Universidad de Costa Rica, 2014.

Los cristales encontrados en los tejidos analizados mostraron una morfología monohidratada. Estos pueden presentar forma romboidal (Figura 2A), prismática, de barra (estiloides
(Figura 2B, 2C) y rafidios (Figura 2D)), pinacoidal (Figura 3A y 3B), agregados esféricos (drusas) $\mathrm{y}$ transiciones entre estos. No se encontraron cristales dihidratados, los cuales muestran forma 
piramidal tetragonal, bipiramidal tetragonal y pinacoidal basal (Hartl et al. 2007).

A pesar de lo anterior, en el género Hylocereus se pueden encontrar cristales con ambos estados de hidratación, los cuales se almacenan en la hipodermis, en el parénquima cortical y en la epidermis (Hartl et al. 2007). En varios genotipos de Hylocereus costaricensis se observó la presencia de cristales de oxalato en el interior de los tejidos con una amplia variabilidad en las morfologías y estados de hidratación de los mismos. La frecuencia de aparición de cada una de las formas fue variable entre genotipos (Viñas 2011).

Cabe destacar que la formación de los cristales no es un proceso al azar. Existen parámetros biofisicoquímicos en el interior del cristal así como fuerzas mecánicas, que podrían afectar fuertemente el desarrollo de estos; además, factores como la temperatura, la presión y la concentración de iones, pueden modificar la morfología y las propiedades del cristal (Franceschi y Horner 1980, Monje y Baran 2002).

Respecto a lo anterior, Franceschi y Nakata (2005) indican que las diferentes formas podrían desarrollarse a partir de especies de oxalato con una química idéntica, pero el ambiente celular regula tipos específicos del cristal así como su función. De acuerdo con esto, y por la naturaleza patológica de este estudio, las morfologías de los cristales encontrados sobre la superficie del tallo de la pitahaya posiblemente variaron en comparación con las conformaciones reportadas por Viñas (2011), debido a que quizás este arreglo molecular permite una mejor cobertura sobre el tejido, sobre todo en tallos con síntomas avanzados.

\section{Análisis mediante difracción de rayos X}

Al comparar el patrón de difracción de rayos $\mathrm{X}$ obtenido de las muestras con la base de datos ICCD (Kabekkodu 2007), se confirmó la presencia de oxalatos de calcio en su forma monohidratada en ambos estados de desarrollo de la enfermedad (flechas azules). La intensidad del patrón de los $\mathrm{Ca}_{2} \mathrm{C}_{2} \mathrm{O}_{4}$ fue relativamente baja probablemente debido a la presencia de la matriz orgánica (picos negros en la frecuencia) que permanece a pesar del tratamiento previo a la extracción (Figuras 4 y 5).

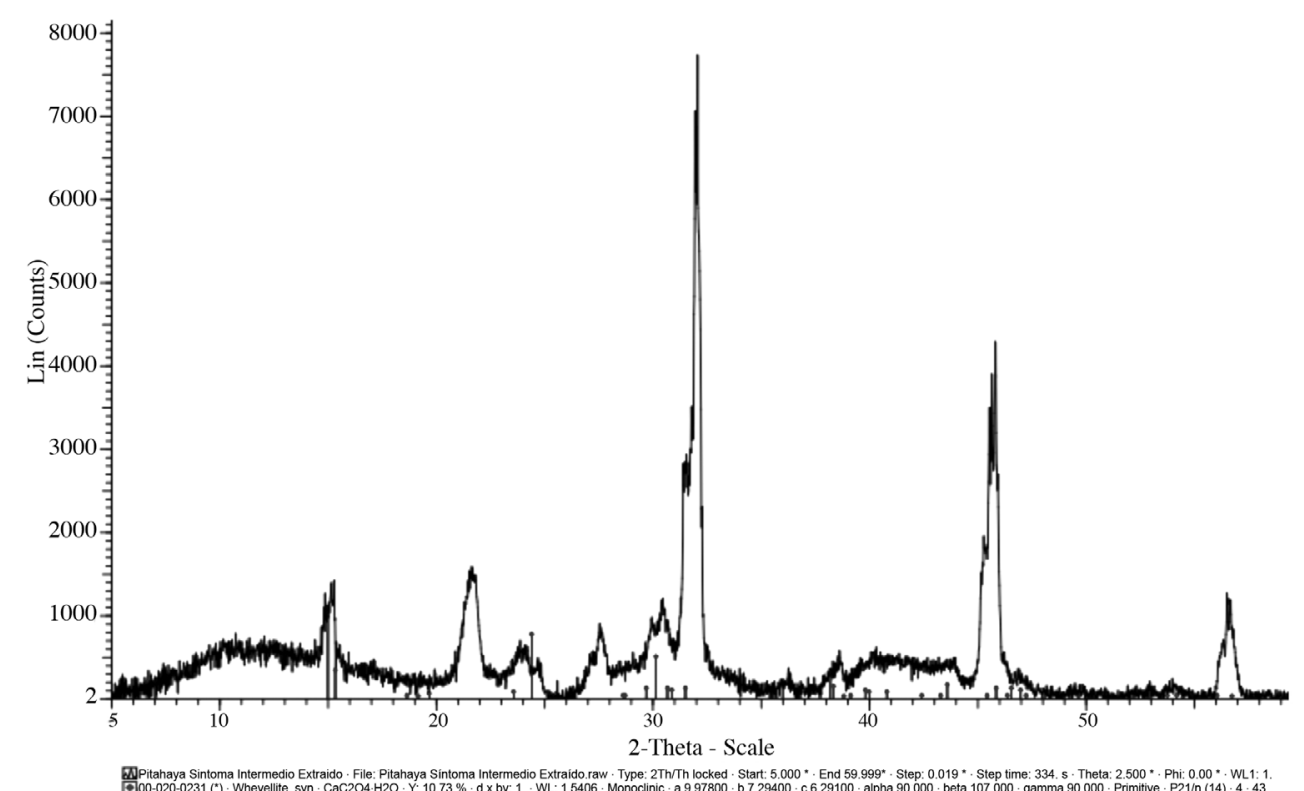

Fig. 4. Patrón de difracción de los oxalatos de calcio presentes en la muestra con síntomas intermedios de la enfermedad mancha blanca en tallos de pitahaya (H. costaricensis). 


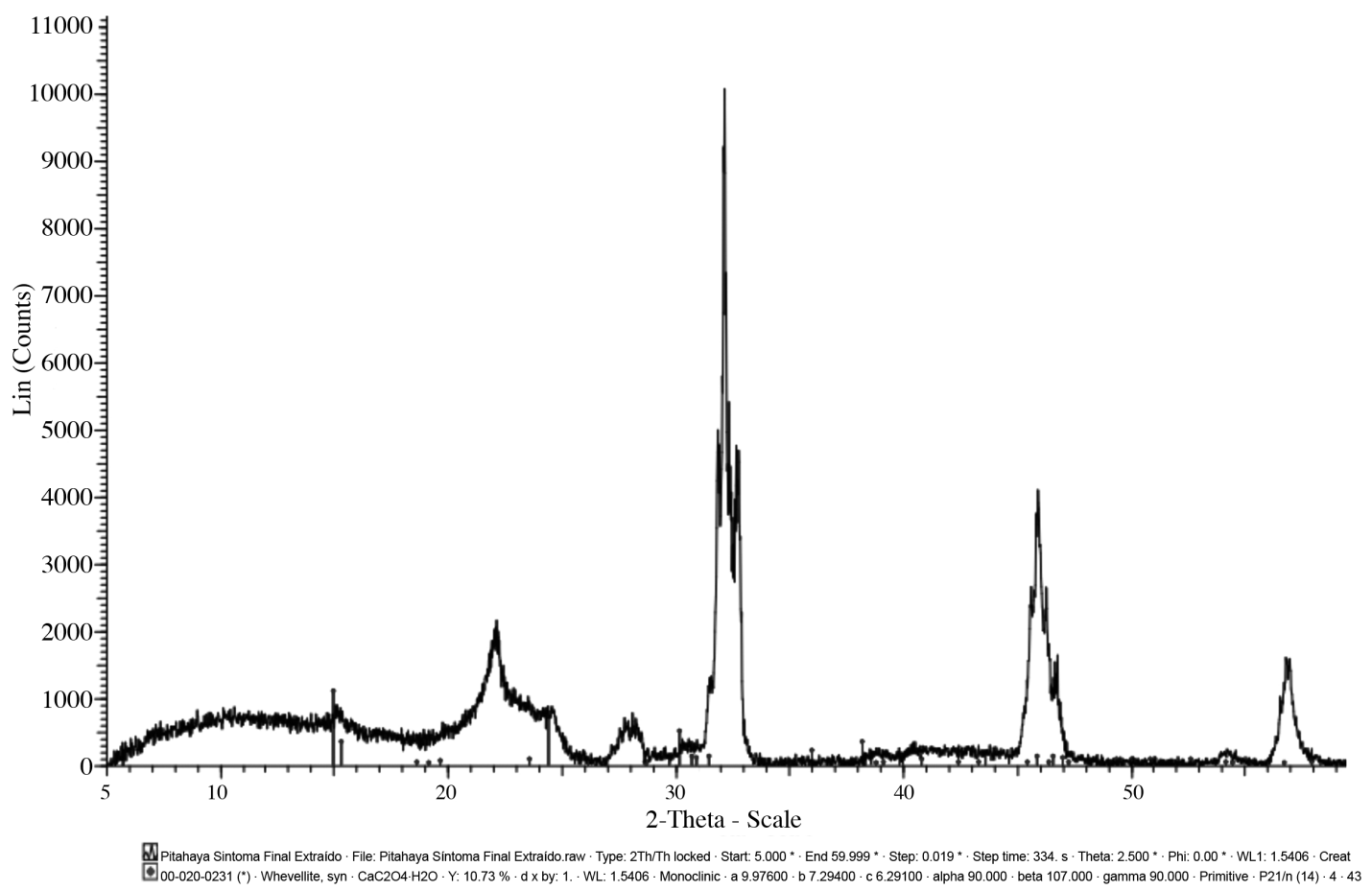

Fig. 5. Patrón de difracción de los oxalatos de calcio presentes en la muestra con síntomas avanzados de la enfermedad mancha blanca en tallos de pitahaya (H. costaricensis).

No se detectó la forma dihidratada de estos, debido a que el equipo utilizado no detecta la wedellita. Lo anterior, no permitió determinar si en el material analizado sólo se puede encontrar conformaciones monohidratadas de los cristales, sin embargo, se presume que sobre la superficie únicamente se presenta whewellita, porque este es el estado de hidratación más estable. Además, los estados dihidratados fácilmente se convierten en estados monohidratados (Monje y Baran 2002, Volk et al. 2002).

Estos resultados respaldan las observaciones de los cristales de este compuesto en el microscopio electrónico y confirman que los oxalatos están asociados al recubrimiento que causa la enfermedad abiótica mancha blanca. Como ya se mencionó, es frecuente encontrar $\mathrm{CaOx}$ en tejidos internos de diferentes cactus (Hartl et al. 2007), por lo que posiblemente los cristales encontrados en $H$. costaricensis se originen en el interior de la planta.
Los oxalatos de calcio están implicados en funciones tales como la regulación del $\mathrm{pH}$ de la célula, percepción de la fuerza de gravedad, soporte mecánico e incluso defensa de la planta contra la herbivoría. Asimismo, pueden estar relacionados con una característica fisiológica particular de las plantas suculentas, la preservación del agua (Franceschi y Horner 1980, Molano 2001, Monje y Baran 2002).

\section{Alteraciones fisiológicas y patológicas}

Debido a que los cristales de oxalato de calcio están asociados al recubrimiento que provoca la enfermedad "mancha blanca", se determinó que estos posiblemente tienen un efecto negativo sobre la fotosíntesis de la planta, debido a que afectan la función clorofílica al impedir la incidencia de luz en los tallos. Asimismo, se altera el intercambio gaseoso, ya que altas concentraciones de $\mathrm{Ca}^{+2}$ promueven el cierre estomático 
(Da Silva et al. 1985). Además, es probable que la red de cristales bloquee los estomas, lo que reduce la captación de dióxido de carbono durante la noche.

Con base en observaciones de campo, en tallos con síntomas muy avanzados, específicamente en los cladodios principales, ocurrió la senescencia completa del tejido, debido a la entrada de patógenos que dejó al descubierto el haz vascular. Lo anterior, se dio primordialmente por la infección y desarrollo de la bacteria $E$. hormaechei, lo cual ocurrió después de que presentara el recubrimiento en la superficie del cladodio (Figura 6).
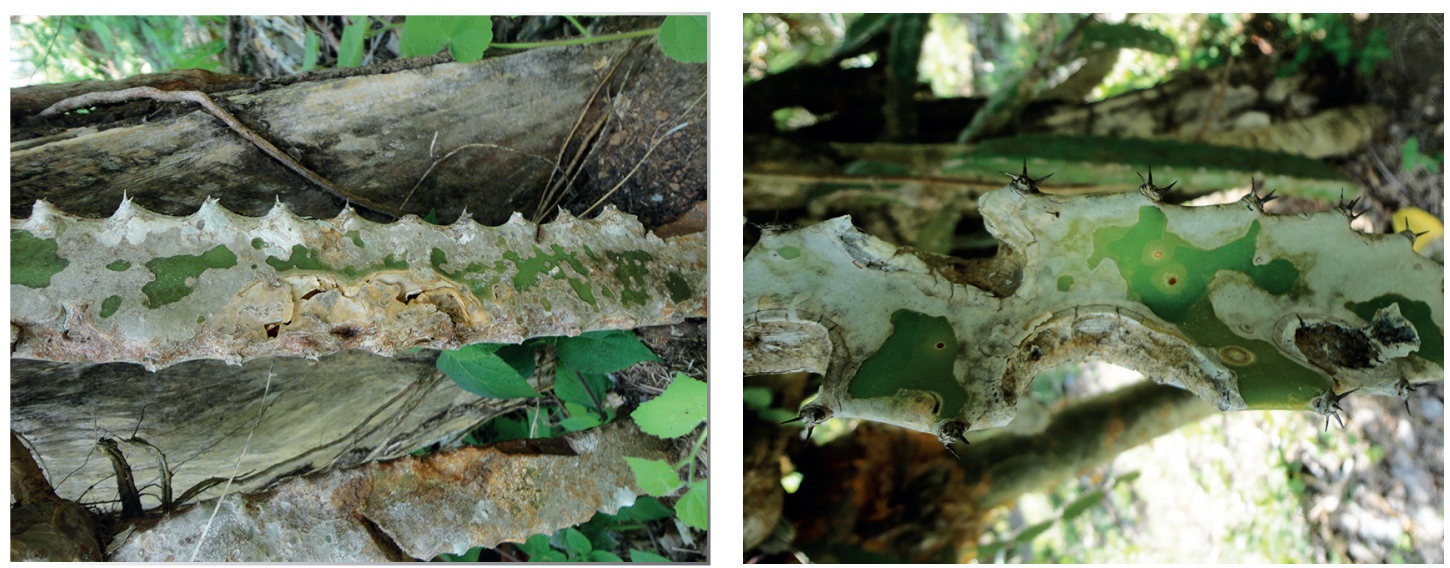

Fig. 6. Asociación entre la bacteria E. hormaechei y los síntomas provocados por el recubrimiento en cladodios de pitahaya (H. costaricensis).

En el área de estudio se observó que todas las plantas presentaban el recubrimiento cálcico, tanto las plantas expuestas a pleno sol como las que se encontraban en sombra, sin embargo, en las primeras el desarrollo del recubrimiento cálcico fue mayor. Horner HT. 2006. Función de los oxalatos de calcio (comunicación personal). Iowa State University, Iowa, Estados Unidos, citado por Hartl et al. (2007), menciona que posiblemente los oxalatos se acumulan dentro de células epidérmicas como un mecanismo de protección contra el exceso de radiación solar.

Con base en lo anterior, es posible que la intensa radiación que reciben estas plantas en el sitio de cultivo sea un factor que estimula la producción e inclusive la liberación de sales cálcicas en respuesta a esta condición, como medida de protección para evitar una reducción en la tasa fotosintética. Respecto a esto, Andrade et al.
(2006) mencionan que en $H$. undatus con una incidencia diaria entre un $36 \%$ y $48 \%$ del flujo de fotones para la fotosíntesis ( $\mu \mathrm{mol} / \mathrm{s})$, la actividad fotosintética fue mayor en un $44 \%$ en comparación con plantas expuestas a una mayor o menor luminosidad.

Cabe indicar que la pitahaya crece en condiciones de sombra media, por lo que la tasa de intercambio gaseoso (TIC) depende de una intensidad lumínica moderada. Por ejemplo, la TIC de plantas de H. undatus expuestas al $70 \%$ de la máxima radiación fotosintéticamente activa (RFA) alcanzó un pico máximo de 1,89 $\mu \mathrm{mol}$ $\mathrm{CO}_{2} \mathrm{~m}^{-2} \mathrm{~s}^{-1}$, mientras que tallos que recibieron $30 \%$ de RFA presentaron un pico máximo de la TIC de 3,89 $\mu \mathrm{mol} \mathrm{CO} \mathrm{m}^{-2} \mathrm{~s}^{-1}$ (Ortiz et al. 1999).

Otro aspecto que podría justificar la formación de este recubrimiento es que las plantas en estudio quizás presentaron un mecanismo de 
exudación de los oxalatos, para evitar intoxicarse con altas concentraciones de calcio, ya que el suelo tiene altos niveles de este elemento (Cuadro 2). Esta premisa se basa en lo mencionado por Ruiz y Mansfield (1994), quienes señalan que la formación y el almacenamiento de los cristales de $\mathrm{Ca}_{2} \mathrm{C}_{2} \mathrm{O}_{4}$ en células epidérmicas, es un mecanismo para regular que altas concentraciones de $\mathrm{Ca}^{+2}$ libre (regulación iónica), presentes en la corriente respiratoria, lleguen a sitios donde podrían ser fisiológicamente perjudiciales.

A pesar de lo anterior, en algunas plantas, por ejemplo Lema minor, se presentan limitaciones en el número de células de almacenamiento (idioblastos) que se pueden producir y en la capacidad máxima de estas para acumular los oxalatos, cuando el medio de crecimiento presenta un alto contenido de calcio. Una vez que los límites se alcanzan, nuevos aumentos de $\mathrm{Ca}^{+2}$ pueden conducir a efectos tóxicos, tales como una disminución en la tasa de crecimiento y senescencia (Mazen et al. 2003). En plantas de $H$. costaricensis, es probable que cuando se alcanzan los límites de almacenamiento de oxalatos, ocurra la liberación de los excedentes a través de las areolas.

Un mecanismo de detoxificación, similar al que posiblemente se presenta en la pitahaya, que fue reportado en árboles de abeto rojo (Picea rubens Sarg.), cuando en el suelo se incrementa el contenido de calcio, que se transloca en mayor cantidad hacia el follaje y se almacena en células de colénquima y de la cutícula, en forma de oxalato (Smith et al. 2009), resultados de este estudio y las observaciones de campo sugieren que la pitahaya posee la capacidad de exudar esta sal, que quizás se da por la eliminación de excedentes de calcio en el interior de la planta, más que por un mecanismo de protección ante una alta radiación solar.

Si la deposición de los oxalatos de calcio sobre la superficie de los tallos es producto de la liberación, a través de la corriente respiratoria, de excesos de calcio presentes en el interior de la planta, es probable que frente a aumentos de temperatura y de intensidad lumínica que fueron las condiciones ambientales bajo las que estuvo la plantación durante la investigación, se desencadene la exudación de los cristales de oxalato, debido al incremento en la tasa respiratoria. Cabe señalar que no existen reportes previos de plantas que presenten alguno de los mecanismos de respuesta descritos ante las condiciones ambientales mencionadas.

En las diferentes zonas productoras de pitahaya alrededor del mundo se reportan una serie de enfermedades bióticas tales como fusariosis (Fusarium oxysporum), antracnosis (Colletotrichum gloeosporioides), chancros (Neoscytalidium dimidiatum), bacteriosis (Erwinia carotovora y Enterobacter cloacae) y virosis (Virus X) (Liou et al. 2000, OIRSA 2000, Palmateer et al. 2007, Wright et al. 2007, Masyahit et al. 2009, Masratul et al. 2013, Sanahuja et al. 2016 ) pero hasta el momento no se han descrito patologías de origen abiótico. Esta investigación establece las bases de la posible causa de la primera enfermedad abiótica que afecta este cultivo.

\section{AGRADECIMIENTOS}

Un especial agradecimiento a la Lic. Marisol Jiménez Chavarría y al Dr. Adrián Pinto Tomás del Centro de Investigación en Estructuras Microscópicas de la Universidad de Costa Rica, por facilitar las instalaciones y el apoyo económico y docente para desarrollar esta investigación.

\section{LITERATURA CITADA}

Andrade, J; Rengifo, E; Ricalde, M; Simá, J; Cervera, J; Vargas, G. 2006. Microambientes de luz, crecimiento y fotosíntesis de la pitahaya (Hylocereus undatus) en un agrosistema de Yucatán, México. Agrociencia 40:687-697.

Atlas, R. 2010. Handbook of microbiological media. Fourth Edition. Washington, USA, ASM Press-CRC Press. $2036 \mathrm{p}$.

Da Silva, D; Hetherington, A; Mansfield, T. 1985. Synergism between calcium ions and abscisic acid in preventing stomatal opening. New Phytologist 100:473-482.

Dembitsky, V; Poovarodom, S; Leontowicz, H; Leontowicz, M; Vearasilp, S; Trakhtenberg, S; Gorinstein, S. 2011 The multiple nutrition properties of some exotic 
fruits: Biological activity and active metabolites. Food Research International 44:1671-1701.

Faheed, F; Mazen, A; Abd Elmohsen, S. 2012. Physiological and ultrastructural studies on calcium oxalate crystal formation in some plants. Turkish Journal of Botany 37:139-152.

Franceschi, V; Horner, H. 1980. Calcium oxalate crystals in plants. Botanical Review 46(4):361-427.

Franceschi, V; Li, L; Zhang, D; Okita, T. 1993. Calsequestrinlike calcium binding protein is expressed in calcium accumulating cells of Pistia stratiotes. Proceedings of the National Academy of Sciences 90:6986-6990.

Franceschi, V; Nakata, P. 2005. Calcium oxalate in plants: formation and function. Plant Biology 56:41-71.

French, ER; Hebert, TT. 1980. Métodos de investigación fitopatológica. San José, Costa Rica, Instituto Interamericano de Ciencias Agrícolas (IICA). 288 p.

Hartl, W; Klapper, H; Barbier, B; Ensikat, H; Dronskowski, R; Müller, P; Ostendorp, G; Tye, A; Bauer, R; Barthlott, W. 2007. Diversity of calcium oxalate crystals in cactaceae. Canadian Journal of Botany 85(5):501-517.

Juárez, A; Livera, M; Sosa, E; Goytia, M; González, V; Bárcena, R. 2012. Composición química de tallos inmaduros de Acanthocereus spp. e Hylocereus undatus (Haw.) Britton \& Rose. Revista Fitotecnia Mexicana 35(2):171-175.

Kabekkodu S. 2007. International Centre for Diffraction Data (ICDD) (en línea). Database. Pensilvania, Estados Unidos. Consultado 24 jun. 2014. Disponible en http://www.icdd.com

Keates, S; Tarlyn, N; Loewus, F; Franceschi, V. 2000. L-Ascorbic acid and L-galactose are sources for oxalic acid and calcium oxalate in Pistia stratiotes. Phytochemistry 53:433-440.

Kostman, T; Tarlyn, N; Loewus, F; Franceschi, V. 2001. Biosynthesis of L-ascorbic acid and conversion of carbons 1 and 2 of $\mathrm{L}$-ascorbic acid to oxalic acid occurs within individual calcium oxalate crystal idioblasts. Plant Physiology 125:634-640.

Liou, M; Hung, C; Liou, R. 2000. First report of Cactus virus $X$ on Hylocereus undatus (Cactaceae) in Taiwan. Plant Disease 85:229.

Masratul, M; Baharuddin, S; Latiffah, Z. 2013. Identification and molecular characterizations of Neoscytalidium dimidiatum causing stem canker of red-fleshed dragon fruit (Hylocereus polyrhizus) in Malaysia. Journal of Phytopathology 161(11-12):841-849.

Masyahit, M; Sijam, K; Awang, Y; Ghazali, M. 2009. First report on bacterial soft rot disease on dragon Fruit (Hylocereus costaricensis) caused by Enterobacter cloacae in peninsular Malaysia. International Journal of Agriculture \& Biology 11:659-666.

Mazen, A; Zhang, D; Franceschi, V. 2003. Calcium oxalate formation in Lemna minor: physiological and ultrastructural aspects of high capacity calcium sequestration. New Phytologist 161:435-448.

Molano, B. 2001. Herbivory and calcium concentrations affect calcium oxalate crystal formation in leaves of Sida (Malvaceae). Annals of Botany 88:387-391.

Monje, P; Baran, E. 2000. First evidences of the bioaccumulation of $\alpha$-quartz in cactaceae. Journal Plant Physiology 157:457-460.

Monje, P; Baran E. 2002. Characterization of calcium oxalates generated as biominerals in cacti. Plant Physiology 128:707-713.

Monje, P; Baran, E. 2010. Characterization of calcium oxalate biominerals in some (non-cactaceae) succulent plant species. Zeitschrift für Naturforschung 65:429-432.

OIRSA. 2000. Manual técnico: Buenas prácticas del cultivo de la pitahaya. Nicaragua, Proyecto Vifinex. 51 p.

Ortiz, Y; Livera, M; Carrillo, J. 1999. Asimilación de $\mathrm{CO}_{2}$ por pitahaya (Hylocereus undatus) en condiciones de campo. Agrociencia 33:165-169.

Palmateer, A; Ploetz, R. 2007. First occurrence of anthracnose caused by Colletotrichum gloeosporioides on pitahaya. Plant Disease 91(5):631.

Retana, K. 2015. Diagnóstico y fluctuación estacional de las enfermedades presentes en el cultivo de la pitahaya (Hylocereus spp.) en Miramar, provincia de Puntarenas. Tesis de maestría. San José, Costa Rica, Universidad de Costa Rica. 110 p.

Ruiz, L; Mansfield, T. 1994. A postulated role for calcium oxalate in the regulation of calcium ions in the vicinity of stomatal guard cells. New Phytologist 127:473-481.

Sanahuja, G; López, P; Palmateer, J. 2016. First Report of Neoscytalidium dimidiatum Penzcausing stem and fruit canker of Hylocereus undatus in Florida. Plant Disease 100(7):1499.

Smith, K; Shortle, W; Connolly, J; Minocha, R; Jellison, J. 2009. Calcium fertilization increases the concentration of calcium in sapwood and calcium oxalate in foliage of red spruce. Environmental and Experimental Botany 67:277-283.

Tenore, G; Novellino, E; Basile, A. 2012. Nutraceutical potential and antioxidant benefits of red pitaya (Hylocereus polyrhizus) extracts. Journal of Functional Foods 4:129-136.

Vargas, M; Centurión, A; Sauri, E; Tamayo, J. 2005. Industrialización de la pitahaya (Hylocereus undatus): una nueva forma de comercialización. Revista Mexicana de Agronegocios 9(16):498-509.

Viñas, M. 2011. Aislamiento purificación y cultivo de protoplasto viables de pitahaya (Hylocereus spp. (Werber) Britton \& Rose). Tesis Lic. San José, Costa Rica, Universidad de Costa Rica 112 p.

Volk, G; Lynch-Holm, V; Kostman, T; Goss, L; Franceschi, R. 2002. The Role of druse and raphide calcium oxalate crystals in tissue calcium regulation in Pistia stratiotes leaves. Plant biology 4:34-45. 
Webb, M. 1999. Cell-mediated crystallization of calcium oxalate in plants. The Plant Cell 11:751-761.
Wright, E; Rivera, M; Ghirlanda, A. 2007. Basal rot of Hylocereus undatus caused by Fusarium oxysporum in Buenos Aires. Plant Disease 91(3):323. 
